\title{
Influência da composição corporal e da idade sobre a densidade óssea em relação aos níveis de atividade física
}

\author{
Influence of body composition and age on bone \\ density in relation to levels of physical activity
}

Thales Boaventura Rachid Nascimento', Maria Fátima Glaner ${ }^{1,2}$, Marina Kanthack Paccini ${ }^{1,3}$

' Grupo de Estudos em Medida e Avaliação, Cineantropometria e Desempenho Humano, Universidade Católica de Brasilia (UCB), Brasilia, DF, Brasil 2 Programa de Pós-Graduação Strictu Sensu em Educação Física, UCB, Brasilia, DF, Brasil ${ }^{3}$ Departamento de Educação Física, Faculdade Estácio de Sá de Ourinhos (Faeso), Ourinhos, SP, Brasil
Correspondência para:

Maria Fátima Glaner

Universidade Católica de Brasília, Programa de Pós-Graduação

Strictu Sensu em Educação Física, Campus I, QS 07, Lote 01, EPCT, Áquas Claras

71966-700 - Brasília, DF, Brasil

mfglaner@ucb.br

mfglaner@gmail.com

Recebido em 28/Set/2008 Aceito em 23/Fev/2009

\begin{abstract}
RESUMO
Objetivo: Verificar em relação ao nível de atividade física (NAF) o quanto cada componente da composição corporal e a idade influenciam na densidade óssea (DO). Métodos: Amostra de 22 homens e 42 mulheres ( 21 a 51 anos) classificados com NAF recomendado e não recomendado para a saúde. A DO do fêmur foi mensurada por meio da DXA. Resultados: Por meio da análise de regressão linear múltipla stepwise $(\mathrm{p} \leq 0,05)$, a idade explicou negativamente a DO em todos os grupos, exceto nos homens com NAF recomendado. Nas mulheres com NAF não recomendado, a gordura relativa (G\%) e a massa gorda (MG) explicaram a DO em todas as regiões de interesse no fêmur, com aumento de até $0,007 \mathrm{~g} / \mathrm{cm}^{2}$ por quilograma de MG. Conclusão: A DO na área deWard sofre maior influência da idade nas mulheres. A MG e a $\mathrm{G} \%$ exercem influência positiva sobre a DO das mulheres com NAF não recomendado. Arq Bras Endocrinol Metab. 2009;53(4):440-5.
\end{abstract}

Descritores

Densidade óssea; composição corporal; atividade física; gordura corporal

\section{ABSTRACT}

Objectives: Verify the physical activity level (PAL), to what extent each body composition component and age influence bone density (BD). Methods: Samples of 22 men and 42 women (21-51 years) classified with recommended and not recommended PAL for health. The femur's BD measured using DXA. Results: Using a analyzing the stepwise linear regression ( $p \leq .05)$, the age explained negatively BD for all groups, except men with recommended PAL. Among women group with not recommended PAL, the relative fat (\%F) and body fat (BF) explained the BD increasing to $0.007 \mathrm{~g} / \mathrm{cm}^{2}$ per BF kilogram in all regions of interest in the femur. Conclusion: The BD in Ward's area has been mostly influenced by age in women. The FM and \%F have positive influence on BD among women with low PAL. Arq Bras Endocrinol Metab. 2009;53(4):440-5.

Keywords

Bone density; body composition; physical activity; body fat

\section{INTRODUÇÃO}

$\Lambda$ densidade óssea (DO) é determinada pela quantiAdade de conteúdo mineral por área de osso, sendo influenciada por vários fatores, como genéticos, massa corporal, deficiência de hormônios sexuais, vitamina D, terapia com glicocorticoides (1), idade $(2,3)$, nível de atividade física (NAF) $(4,5)$, fumo (2), ingestão de álcool (6), proteínas, fósforo, vitamina K e cálcio (7).
Estudos apontam a eficácia do exercício físico na preservação, na manutenção e/ou no acréscimo de massa e DO em várias idades $(8,9)$. O exercício físico se destaca por ser o único meio de intervenção que pode aumentar potencialmente a força muscular e a massa óssea (10). Seus benefícios sobre a DO são mantidos por longos períodos, mesmo após a redução dos níveis de atividade física, tanto em mulheres (11) quanto em homens (12). 
Assim como o exercício físico, a massa corporal (MC) apresenta correlação positiva com a $\mathrm{DO}(13,14)$. Porém, existem controvérsias acerca de qual componente da MC explica melhor a DO, a massa corporal magra $(\mathrm{MCM})(3,15)$ ou a massa gorda $(\mathrm{MG})(16,17)$.

Em adultos, o avanço da idade influencia em ambos os sexos a redução da DO e do conteúdo ósseo corporal $(2,3,14,18)$, nas mulheres a MCM e a MG e nos homens a MCM (18).

Em razão de indivíduos com maiores níveis de atividade física apresentarem maior $\operatorname{MCM}(3,19)$ e DO $(4,5,19)$, além de menor MG $(2,19)$, esperava-se que existisse alteração na contribuição de cada um dos componentes da MC (MG e MCM) sobre a DO.

Diante do exposto e em razão de a baixa $\mathrm{DO}$ aumentar os riscos de fraturas ósseas, são gerados elevados índices de morbimortalidade e gastos econômicos (1). Portanto, é relevante identificar os fatores que mais influenciam a DO em uma amostra brasileira.

Nesse sentido, o presente estudo tem como objetivo verificar, em relação ao NAF, o quanto a composição corporal e a idade influenciam a DO de homens e mulheres.

\section{MÉTODOS}

\section{Amostra}

A amostra foi composta por 22 homens e 42 mulheres, com idades entre 22 e 59 anos, os quais declararam não ter conhecimento de nenhuma doença. A amostra foi contatada, por meio de panfletos, em hospitais do Distrito Federal e na própria Universidade onde o estudo foi conduzido.

Os critérios de exclusão foram: possuir próteses, pinos de metal no corpo e gravidez.

\section{Coleta de dados}

O estudo foi aprovado pelo Comitê de Ética institucional (processo $n^{\circ} 36 / 2007$ ). Antes de assinarem o termo de consentimento livre e esclarecido, os voluntários foram informados dos objetivos, procedimentos e possíveis desconfortos, sendo-lhes permitida a desistência a qualquer momento, sem sofrer qualquer tipo de dano.

Para atender ao objetivo do estudo, foram mensuradas as seguintes variáveis: $\mathrm{MC}$, estatura, gordura relativa $(\mathrm{G} \%)$, DO na região do fêmur e NAF. A idade foi informada pelo voluntário.

A medida da MC foi feita em uma balança Filizola com escala de variação de $0,1 \mathrm{~kg}$ e com capacidade para até $150 \mathrm{~kg}$. O voluntário avaliado permaneceu em pé, de frente para a escala, pés afastados lateralmente, corpo ereto e com o olhar fixo à frente. A medida da estatura foi feita com o estadiômetro que vem acoplado na balança usada para medir a MC. Para tanto, o voluntário permaneceu descalço em posição ortostática, com os pés unidos, cabeça orientada paralela ao solo e em apneia inspiratória.

A DO e a G\% foram mensuradas pela técnica de absortometria de raios X de dupla energia (DXA) (Lunar, DPX-IQ, software v. $4.7 \mathrm{e})$.

A DO foi medida no fêmur proximal nas regiões de interesse no colo, eixo, inteiro, trocanter e área de Ward. Para tal medida, o voluntário permaneceu deitado em decúbito dorsal e imóvel sobre a mesa do equipamento, pés imobilizados e posicionados por um suporte e as palmas das mãos cruzadas sobre o tórax.

A G\% foi medida pelo modo de análise de corpo inteiro. O voluntário permaneceu em decúbito dorsal, com os pés unidos e braços estendidos ao longo do tronco.

Para garantir a qualidade dos dados, as medidas e as análises dos exames da DO e da G\% foram feitas por um único avaliador com larga experiência nesta atividade. As calibrações diárias e semanais foram realizadas conforme recomendações do fabricante do aparelho, seguindo rigorosamente o protocolo descrito no manual que acompanha o referido equipamento.

A partir da $\mathrm{G} \%$ fornecida pela DXA foi calculada a MG: $M G=M C(100 / G \%)$. A MCM foi obtida da subtração entre a $\mathrm{MC}$ e a $\mathrm{MG}(\mathrm{MCM}=\mathrm{MC}-\mathrm{MG})$.

A estimativa do NAF foi realizada por meio do Questionário de Atividades Físicas Habituais, traduzido e adaptado por Nahas (20). Os sujeitos foram classificados em quatro categorias. Os classificados como inativos e pouco ativos foram considerados com NAF não recomendado para a saúde, ao passo que os classificados de moderadamente ativos a muito ativos foram considerados com NAF recomendado para a saúde.

\section{Análise estatística}

Para a caracterização da amostra nas variáveis mensuradas, foi utilizada a estatística descritiva. A análise de regressão linear múltipla stepwise $(\mathrm{p} \leq 0,05)$ foi usada para verificar a contribuição das variáveis independentes (MC, MG, MCM, G\% e idade) sobre a DO (variável dependente) nas regiões de interesse no fêmur. $\mathrm{O}$ coeficiente de correlação de Pearson foi usado para verificar o relacionamento entre as variáveis independentes com a variável dependente. Para a análise estatística, foi utilizado o SPSS (Statistical Package for the Social Sciences) para Windows versão 14.0, com licença de uso. 


\section{RESULTADOS}

Tanto as mulheres quanto os homens classificados com NAF recomendado apresentaram maior DO, e menor $\mathrm{MG}$, em relação aos respectivos pares por sexo. As mulheres com NAF recomendado apresentaram menor MC e MCM em relação ao seu respectivo par. A idade apresenta maior variação em ambos os sexos com NAF não recomendado (Tabela 1 ).

$\mathrm{Na}$ Tabela 2, são apresentados os resultados da análise de regressão linear múltipla $(\mathrm{p} \leq 0,05)$. No grupo de homens com NAF não recomendado para a saúde e de mulheres com NAF recomendado, somente a idade explicou a DO na área de Ward em 43\% e 58\%, respectivamente.

No grupo de mulheres com NAF não recomendado para a saúde, as variáveis idade e MG, juntas, explicaram em $31 \%$ a DO na área de Ward e a idade sozinha explicou em 17\%. Para as regiões de interesse no trocanter, eixo e inteiro do fềmur, a $\mathrm{MG}$ explicou, respectivamente, em $15 \%, 23 \%$ e $20 \%$ a DO. A região do colo foi explicada em $20 \%$ pela $\mathrm{G} \%$.

No grupo de homens com NAF recomendado para a saúde, nenhuma das variáveis explicou a DO nas regiões de interesse no fềmur.

Nenhuma das variáveis mostrou correlação significativa com a DO das regiões de interesse no fêmur, no grupo de homens com NAF recomendado para a saúde (Tabela 3), enquanto para o grupo de homens com NAF não recomendado houve correlação inversa e significativa $(\mathrm{p} \leq 0,05)$, porém de magnitude moderada da idade com a DO $(r=-0,498)$ no colo do fêmur e área de Ward $(r=-0,654)$.
Para o grupo das mulheres com NAF recomendado para a saúde, a DO na área de Ward apresentou boa correlação $(r=-0,760 ; \mathrm{p} \leq 0,05)$ inversa com a idade. Para o grupo das mulheres com NAF não recomendado para a saúde, houve correlação $(\mathrm{p} \leq 0,05)$ positiva, de magnitude razoável entre a G\% e a DO de todas as regiões de interesse no fềmur (colo $\mathrm{r}=0,45 \mathrm{l}$; Ward $\mathrm{r}=0,307$; eixo $r=0,394$; inteiro $r=0,282$; trocanter $r=0,356$ ); entre a MG e a DO no colo $(r=0,446)$, eixo $(r=0,488)$, inteiro $(r=0,450)$ e trocanter $(r=0,390)$ e entre a MC com a DO do eixo $(r=0,337)$ e na região inteira do fềmur $(r=0,291)$. Uma correlação $(p \leq 0,05)$ inversa entre a idade e a DO na área de Ward $(r=-0,417)$.

\begin{tabular}{|c|c|c|c|}
\hline Regressão & $\mathbf{R}$ & $\mathbf{R}^{2}$ & p \\
\hline \multicolumn{4}{|l|}{ NAF não recomendado - Homens } \\
\hline DO Ward = 1,226 - 0,007 (idade) & 0654 & 0,427 & 0,011 \\
\hline \multicolumn{4}{|l|}{ NAF recomendado - Mulheres } \\
\hline D0 Ward = 1,441 - 0,011 (idade) & 0,760 & 0,577 & 0,047 \\
\hline \multicolumn{4}{|l|}{ NAF não recomendado - Mulheres } \\
\hline $\mathrm{DO}$ do colo $=0,814+0,004(\mathrm{G} \%)$ & 0,451 & 0,203 & 0,003 \\
\hline DO Ward = 1,101 - 0,006 (idade) & 0,417 & 0,174 & 0,006 \\
\hline D0 Ward $=0,999-0,008$ (idade) $+0,007$ (MG) & 0,558 & 0,312 & 0,008 \\
\hline D0 trocanter $=0,731+0,005(\mathrm{MG})$ & 0,390 & 0,152 & 0,011 \\
\hline D0 eixo $=1,029+0,007(\mathrm{MG})$ & 0,488 & 0,239 & 0,001 \\
\hline D0 inteiro $=0,884+0,006(\mathrm{MG})$ & 0,450 & 0,202 & 0,003 \\
\hline
\end{tabular}

R: coeficiente de correlação múltipla; R2: coeficiente de determinação; p: nivel de significância; NAF: nível de atividade física; DO: densidade óssea; G\%: gordura relativa; MG: massa gorda.

\begin{tabular}{|c|c|c|c|c|}
\hline \multirow[b]{2}{*}{ Variáveis } & \multicolumn{2}{|c|}{ Mulheres } & \multicolumn{2}{|c|}{ Homens } \\
\hline & $\begin{array}{l}\text { NAF recomendado } \\
(n=7)\end{array}$ & $\begin{array}{l}\text { NAF não recomendado } \\
(\mathrm{n}=35)\end{array}$ & $\begin{array}{l}\text { NAF recomendado } \\
(\mathrm{n}=8)\end{array}$ & $\begin{array}{l}\text { NAF não recomendado } \\
(\mathrm{n}=14)\end{array}$ \\
\hline Idade (anos) & $47,4 \pm 11,0$ & $40,3 \pm 12,1$ & $25,5 \pm 2,3$ & $37,6 \pm 12,3$ \\
\hline Massa corporal (kg) & $60,2 \pm 9,9$ & $65,3 \pm 14,2$ & $79,0 \pm 9,6$ & $78,0 \pm 10,9$ \\
\hline Estatura (cm) & $154,0 \pm 7,5$ & $158,6 \pm 6,8$ & $180,5 \pm 6,8$ & $171,3 \pm 7,8$ \\
\hline Gordura (\%) & $35,9 \pm 6,2$ & $38,0 \pm 15,4$ & $13,1 \pm 3,9$ & $22,2 \pm 5,5$ \\
\hline MCM (kg) & $38,2 \pm 4,1$ & $40,2 \pm 10,3$ & $68,5 \pm 8,3$ & $60,2 \pm 5,4$ \\
\hline$M G(k g)$ & $22,0 \pm 6,9$ & $25,1 \pm 10,2$ & $10,4 \pm 3,5$ & $17,8 \pm 6,3$ \\
\hline D0 colo fêmur (g/cm²) & $1,046 \pm 0,121$ & $0,971 \pm 0,141$ & $1,242 \pm 0,134$ & $1,085 \pm 0,119$ \\
\hline D0 área Ward (g/cm²) & $0,899 \pm 0,165$ & $0,852 \pm 0,177$ & $1,124 \pm 0,148$ & $0,949 \pm 0,138$ \\
\hline D0 eixo $\left(\mathrm{g} / \mathrm{cm}^{2}\right)$ & $1,265 \pm 0,185$ & $1,214 \pm 0,154$ & $1,410 \pm 0,191$ & $1,352 \pm 0,100$ \\
\hline D0 trocanter $\left(\mathrm{g} / \mathrm{cm}^{2}\right)$ & $0,911 \pm 0,123$ & $0,855 \pm 0,129$ & $1,078 \pm 0,174$ & $0,948 \pm 0,104$ \\
\hline D0 inteiro (g/cm²) & $1,084 \pm 0,135$ & $1,034 \pm 0,136$ & $1,241 \pm 0,165$ & $1,131 \pm 0,092$ \\
\hline
\end{tabular}

NAF: nível de atividade física; MCM: massa corporal magra; MG: massa gorda; DO: densidade óssea. 
Tabela 3. Correlação de Pearson entre a densidade óssea das regiões de interesse no fêmur e as variáveis gordura relativa, massa gorda, massa corporal magra, massa corporal e idade

\begin{tabular}{|c|c|c|c|c|c|}
\hline Regiões & G\% & MG & MCM & MC & Idade \\
\hline \multicolumn{6}{|c|}{ NAF recomendado - Homens } \\
\hline Colo & - & 0,085 & 0,077 & 0,097 & $-0,027$ \\
\hline Ward & 0,103 & 0,143 & 0,139 & 0,172 & $-0,199$ \\
\hline Eixo & $-0,071$ & $-0,075$ & 0,002 & $-0,026$ & $-0,422$ \\
\hline Inteiro & 0,049 & 0,054 & 0,040 & 0,054 & $-0,309$ \\
\hline Trocanter & 0,157 & 0,168 & 0,069 & 0,121 & $-0,227$ \\
\hline \multicolumn{6}{|c|}{ NAF não recomendado - Homens } \\
\hline Colo & $-0,022$ & $-0,040$ & $-0,020$ & $-0,038$ & $-0,498^{*}$ \\
\hline Ward & $-0,240$ & $-0,226$ & $-0,085$ & $-0,173$ & $-0,654^{*}$ \\
\hline Eixo & 0,039 & $-0,024$ & $-0,142$ & $-0,084$ & $-0,236$ \\
\hline Inteiro & $-0,144$ & $-0,152$ & $-0,159$ & $-0,167$ & $-0,189$ \\
\hline Trocanter & $-0,208$ & $-0,227$ & $-0,173$ & $-0,217$ & 0,044 \\
\hline \multicolumn{6}{|c|}{ NAF recomendado - Mulheres } \\
\hline Colo & $-0,334$ & $-0,333$ & $-0,083$ & $-0,268$ & $-0,573$ \\
\hline Ward & $-0,435$ & $-0,471$ & $-0,218$ & $-0,421$ & $-0,760^{\star}$ \\
\hline Eixo & $-0,281$ & $-0,283$ & $-0,172$ & $-0,270$ & $-0,278$ \\
\hline Inteiro & $-0,233$ & $-0,218$ & $-0,094$ & $-0,191$ & $-0,263$ \\
\hline Trocanter & $-0,122$ & $-0,107$ & $-0,090$ & $-0,113$ & $-0,057$ \\
\hline \multicolumn{6}{|c|}{ NAF não recomendado - Mulheres } \\
\hline Colo & $0,451^{*}$ & $0,446^{*}$ & $-0,160$ & 0,204 & $-0,177$ \\
\hline Ward & $0,307^{\star}$ & 0,231 & $-0,186$ & 0,031 & $-0,417^{*}$ \\
\hline Eixo & $0,394^{*}$ & $0,488^{*}$ & $-0,020$ & $0,337^{*}$ & 0,134 \\
\hline Inteiro & $0,282^{*}$ & $0,450^{*}$ & $-0,045$ & $0,291^{*}$ & 0,046 \\
\hline Trocanter & $0,356^{*}$ & $0,390^{*}$ & $-0,062$ & 0,235 & 0,090 \\
\hline
\end{tabular}

*: $p \leq 0,05 ;$ G\%: gordura relativa; MG: massa gorda; MCM: massa corporal magra; MC: massa corporal; NAF: nível de atividade física.

\section{DISCUSSÃO}

No presente estudo, os resultados indicam que no grupo de homens com NAF não recomendado e em ambos os grupos de mulheres somente a idade explicou a DO do fêmur, na área de Ward, principalmente no grupo das mulheres com NAF recomendado. Isso demonstra que, com o aumento da idade, há redução na DO de até $0,011 \mathrm{~g} / \mathrm{cm}^{2}$ por ano, o que ratifica os resultados encontrados em outros estudos $(2,3)$.

A redução da DO em função da idade, verificada somente na área de Ward, possivelmente ocorra por tal região ser composta em grande parte por osso trabecular, o qual apresenta perda óssea mais acentuada em relação ao osso cortical (2l). Porém, outros motivos contribuem para tal redução da $\mathrm{DO}$, como a redução dos níveis de atividade física em relação à idade (3) e a menor capacidade da pele em produzir vitamina $\mathrm{D}$, por meio da radiação solar (1).

Entretanto, no grupo de homens com NAF recomendado, a idade não explicou a DO em nenhuma das regiões de interesse no fềmur. Possivelmente, isso tenha ocorrido pelo reduzido número da amostra e pela homogeneidade apresentada pelo grupo, o que os caracteriza ainda em período de aquisição de massa óssea (22).

Um maior efeito deletério da idade sobre a DO encontrado no grupo das mulheres com NAF recomendado em comparação ao grupo com NAF não recomendado chama a atenção, pois era previsível que maiores níveis de atividade física pudessem reduzir a perda da DO ocasionada pela idade. Tal achado pode ser explicado pela influência dos fatores genéticos sobre a DO, pelo pequeno número da amostra com NAF recomendado e pelos hábitos alimentares dos participantes, tendo em vista que um déficit na ingesta de nutrientes essenciais para a manutenção da DO, tal como a vitamina $\mathrm{D}$, cálcio e/ou proteínas, mesmo nas mulheres com bom NAF, pode potencializar a perda de DO ocasionada pela idade.

No grupo de mulheres com NAF não recomendado, a $\mathrm{G} \%$ e a $\mathrm{MG}$ explicaram a DO em todas as regiões de interesse no fềmur, com aumento da DO de até 0,007 $\mathrm{g} / \mathrm{cm}^{2}$ por quilo de MG. Isso corrobora os dados observados por Douchi e cols. (19), os quais encontraram correlação positiva e significativa entre a $\mathrm{MG}$ e a DO na coluna lombar, somente no grupo de mulheres sedentárias. Possivelmente, esse efeito benéfico da MG sobre a DO seja mediado pela sobrecarga mecânica gerada pela $\mathrm{MG}$, conversão de andrógenos para estrógenos no tecido adiposo por meio do processo de aromatização (23) e pela maior força muscular aplicada sobre o sistema ósseo para mover a resistência adicional proveniente da MG (24).

Não obstante o aumento da DO ser concomitante ao da MG, é claro na literatura (25) que pessoas com maior acúmulo de $\mathrm{G} \%$ estão mais propensas às doenças crônicas degenerativas, como as cardíacas, o diabetes e a hipertensão.

No grupo de mulheres com NAF não recomendado, a MG explicou por volta de $20 \%$ a DO do fêmur inteiro. Esse resultado é similar ao encontrado (23\%) por Douchi e cols. (19), em mulheres inativas, porém na coluna. Em outro estudo (15), foi avaliada a DO na região da coluna em homens e mulheres, porquanto a MG apresentou significância somente nas mulheres. Ao passo que, no estudo de Gjesdal e cols. (2), em que foi 
analisada a DO do colo do fềmur, a MG apresentou significância positiva, gerando aumento na DO de 0,001 $\mathrm{g} / \mathrm{cm}^{2} \mathrm{em}$ homens e $0,002 \mathrm{~g} / \mathrm{cm}^{2}$ em mulheres, por quilo de $\mathrm{MG}$.

Diante desses resultados $(2,15)$, juntamente com os encontrados no presente estudo, percebe-se que a $\mathrm{MG}$ exerce maior influência $(\mathrm{p} \leq 0,05)$ em várias regiões ósseas das mulheres. Já para os homens, parece que a $\mathrm{MG}$ parece exercer efeitos significativos $(\mathrm{p} \leq 0,05)$ e de menor magnitude somente em algumas regiões ósseas.

Mesmo a MCM sendo considerada o principal determinante da DO $(3,15)$, em razão do estresse mecânico mediado pela ação da gravidade e pela contração muscular sobre os ossos (15) e de a MC apresentar correlação positiva com a DO $(13,14)$, nenhuma dessas variáveis explicou a DO em nenhum dos grupos do presente estudo. Possivelmente, isso se deve ao pequeno número das amostras, em especial, nos grupos com NAF recomendado.

O presente estudo apresenta algumas limitações. Uma delas é o reduzido número de voluntários com NAF recomendado, pois isso pode ter contribuído para que não fossem encontradas evidências sobre a influência da composição corporal sobre a DO. Outra limitação é inerente ao questionário, cujos resultados podem ser superestimados ou subestimados por falta de entendimento e/ou capacidade de recordar dos sujeitos (26). No entanto, o questionário usado tem apresentado boa concordância com o consumo de oxigênio, em amostras brasileiras (27).

A não investigação dos hábitos alimentares e do NAF em período anterior ao início do estudo também pode ser considerada limitação. O NAF dos participantes no passado poderia gerar interpretação errônea acerca de qual dos componentes da composição corporal, MG ou MCM, é o maior determinante da DO. Tal erro poderia ocorrer em decorrência de os efeitos do exercício físico sobre a DO serem mantidos por longos períodos, mesmo após a interrupção de sua prática $(11,12)$. Com isso, os indivíduos poderiam apresentar DO elevada por causa do alto NAF no passado, embora apresentem elevada MG e diminuída MCM, possivelmente pelo seu baixo NAF no presente, podendo, desse modo, ser creditada erroneamente maior influência da MG sobre a DO.

Consideradas as limitações inerentes a qualquer estudo, os resultados obtidos possibilitam identificar a importância da composição corporal e do NAF para a manutenção e/ou o aumento da DO, em uma amostra brasileira.
Com base nos resultados obtidos, conclui-se que, em ambos os sexos, a DO da área de Ward sofre maior influência da idade, com efeito mais acentuado nas mulheres. Das variáveis da composição corporal, a MG e a G\% são as únicas que explicam a $\mathrm{DO}$ do fềmur nas mulheres com NAF não recomendado, exercendo efeitos positivos e de magnitudes variáveis nas regiões de interesse no fêmur. A MCM e a MC não explicaram a DO do fêmur nas mulheres e nos homens, independentemente do NAF.

Declaração: os autores declaram não haver conflitos de interesse científico neste estudo.

\section{REFERÊNCIAS}

1. World Health Organization. Prevention and management of osteoporosis: Report of a WHO scientific group. Geneva; 2003.

2. Gjesdal CG, Halse JI, Eide GE, Brun JG, Tell GS. Impact of lean mass and fat mass on bone mineral density: The Hordaland Health Study. Maturitas. 2008;59:191-200.

3. Proctor DN, Melton LJ, Khosla S, Crowson CS, O'Connor MK, Riggs BL. Relative influence of physical activity, muscle mass and strength on bone density. Osteoporos Int. 2000;11:944-52.

4. Egan E, ReillyT, Giacomoni M, Redmond L,Turner C. Bone mineral density among female sports participants. Bone. 2006;38:227-33.

5. Andreoli A, Monteleone M, Loan MV, Promenzio L, Tarantino U, De Lorenzo A. Effects of different sports on bone density and muscle mass in highly trained athletes. Med Sci Sports Exerc. 2001;33:507-11.

6. Rapuri PB, Gallagher JC, Balhorn KE, Ryschon KL. Alcohol intake and bone metabolism in elderly women. Am J Clin Nutr. 2000;72:1206-13.

7. Whiting SW, Boyle JL, Thompson A, Mirwald RL, Faulkner RA. Dietary protein, phosphorus and potassium are beneficial to bone mineral density in adult men consuming adequate dietary calcium. J Am Coll Nutr. 2002;21:402-9.

8. Fuchs RK, Bauer JJ, Snow CM. Jumping improves hip and lumbar spine bone mass in prepubescent children: a randomized controlled trial. J Bone Miner Res. 2001;16:148-56.

9. Borer KT, Fogleman K, Gross M, La New JM, Dengel D. Walking intensity for postmenopausal bone mineral preservation and accrual. Bone. 2007;41:713-21.

10. Bloomfield SA, Wendy K, Little KD. Physical activity and bone health. Med Sci Sports Exerc. 2004;36:1985-96.

11. Moser DC, Melo SIL, Santos SG. Influência da atividade física sobre a massa óssea de mulheres. Rev Bras Cineantropom Desempenho Hum. 2004;6:46-53.

12. Nordström A, OlssonT, Nordström P. Sustained benefits from previous physical activity on bone mineral density in males. J Clin Endocrinol Metab. 2006;91:2600-04.

13. Chaves LM, Gomes L, Oliveira RJ, Marques MB. Relação entre variáveis da composição corporal e densidade mineral óssea em mulheres idosas. Rev Bras Med Esporte. 2005;11:352-6.

14. Zanette E, Stringari FF, Machado F, Marroni BJ, Ng DPK, Canani LH. Avaliação do diagnóstico densitométrico de osteoporose/ osteopenia conforme o sítio ósseo. Arq Bras Endocrinol Metab. 2003;47:30-6.

15. Bakker I, Twisk JWR, Mechelen WV, Kemper HCG. Fat-free body mass is the most important body composition determinant of 10 - 
yr longitudinal development of lumbar bone in adult men and women. J Clin Endocrinol Metab. 2003;88:2607-13.

16. Richards JB, Valdes AM, Burling K, Perks UC, Spector TD. Serum adiponectin and bone mineral density in women. J Clin Endocrinol Metab. 2007;92:1517-23.

17. Lenchik L, Register TC, Hsu FC, Lohman K, Nicklas BJ, Freedman $\mathrm{BI}$, et al. Adiponectin as a novel determinant of bone mineral density and visceral fat. Bone. 2003;33:646-51.

18. Lim S, Joung H, Shin CS, Lee HK, Kim KS, Shin EK, et al. Body composition changes with age have gender-specific impacts on bone mineral density. Bone. 2004;35:792-8.

19. Douchi T, Matsuo T, Uto H, Kuwahata T, Oki T, Nagata, Y. Lean body mass and bone mineral density in physically exercising postmenopausal women. Maturitas. 2003;45:185-90.

20. Nahas MV. Atividade física, saúde e qualidade de vida: conceitos e sugestões para um estilo de vida ativo. $3^{a}$ ed. Londrina: Midiograf; 2003.

21. Agarwal M, Camacho P. Bone densitometry. Interpretation and pitfalls. Postgrad Med. 2006;119:17-23.
22. Pinto Neto AM, Soares A, Urbanetz AA, Souza ACA, Ferrari AEM, Amaral B, et al. Consenso brasileiro de osteoporose. Rev Bras Reumatol. 2002;46:343-54.

23. Klein KO, Larmore KA, De Lancey E, Brown JM, Considine RV, Hassink SG. Effect of obesity on estradiol level, and its relationship to leptin, bone maturation, and bone mineral density in children. J Clin Endocrinol Metab. 1998;83:3469-75.

24. Petit MA, BeckTJ, Shults J, Zemel BS, Foster BJ, Leonard MB. Proximal femur bone geometry is appropriately adapted to lean mass in overweight children and adolescents. Bone. 2005;36:568-76.

25. Burke GL, Bertoni AG, Shea S, Tracy R, Watson KE, Blumenthal RS, et al. The impact of obesity on cardiovascular disease risk factors and subclinical vascular disease. Arch Intern Med. 2008;168:928-35.

26. Glaner MF Nível de atividade física e aptidão física relacionada à saúde em rapazes rurais e urbanos. Rev Bras Educ Fis Esp. 2002;16:76-85.

27. Glaner MF. Concordância de questionários de atividade física com a aptidão cardiorrespiratória. Rev Bras Cineantropom Desempenho Hum. 2007;9:61-6. 